\title{
Cardiac autonomic neuropathy in nonobese young adults with type 1 diabetes
}

Hwa Young Kim, MD', Hae Woon Jung, $\mathrm{MD}^{2}$, Young Ah Lee, MD, PhD', Choong Ho Shin, MD, PhD ${ }^{3}$, Sei Won Yang, MD, PhD ${ }^{3}$

${ }^{1}$ Department of Pediatrics, Kangwon National University Hospital, Kangwon National University School of Medicine, Chuncheon, Korea

${ }^{2}$ Department of Pediatrics, Kyung Hee University Medical Center, Seoul, Korea ${ }^{3}$ Department of Pediatrics, Seoul National University College of Medicine, Seoul, Korea
Received: 14 November, 2018

Revised: 5 January, 2019

Accepted: 16 January, 2019

Address for correspondence:

Young Ah Lee, MD, PhD

Division of Endocrinology and Metabolism, Department of Pediatrics, Seoul National University Children's Hospital, Seoul National University College of Medicine, 101 Daehak-ro, Jongno-gu, Seoul 03080, Korea

Tel: +82-2-2072-2308

Fax: +82-2-743-3455

E-mail:nina337@snu.ac.kr

https://orcid.org/0000-0001-91791247
Purpose: The aim of this study was to evaluate the prevalence and risk factors for cardiac autonomic neuropathy (CAN) in nonobese nonobese young type 1 diabetes mellitus (T1DM) patients without micro- or macrovascular complications.

Methods: CAN was assessed in 95 patients with T1DM, aged 18-29 years, using standard cardiovascular reflex tests - heart rate response to deep breathing, standing, and the Valsalva maneuver and blood pressure response to standing. Furthermore, power spectral analyses of overall heart rate variability (HRV), standard deviation of NN intervals (SDNN), and total power (TP) were tested with DiCAN. CAN was defined as abnormal results for at least 1 of the 4 cardiovascular reflex tests.

Results: The prevalence of CAN was $12.6 \%$. The frequency of one and 2 abnormal reflex tests was $10.5 \%$ and $2.1 \%$, respectively. No significant differences were observed in age, sex, mean hemoglobin $\mathrm{A}_{1 \mathrm{c}}\left(\mathrm{HbA}_{1 c}\right)$ level, and duration of diabetes with respect to presence of CAN. Patients with CAN exhibited lower overall HRV parameters (SDNN and TP) compared with those without CAN even though there was no statistical significance. In multivariable analyses, higher mean $\mathrm{HbA}_{1 c}$ level was significantly associated with lower overall HRV $(\beta=-44.42, P=0.002$ for SDNN and $\beta=-2.82, P<0.001$ for TP).

Conclusion: CAN can be detected in $12.6 \%$ of young adult T1DM patients even without other micro- or macrovascular complications. Glycemic control is the main determinant to maintain overall HRV and prevent CAN.

Keywords: Autonomic nervous system diseases, Type 1 diabetes, Prevalence, Risk factors, Young adult, Korea

\section{Introduction}

Diabetic cardiac autonomic neuropathy (CAN) is caused by damage to the autonomic nerve fibers that innervate the heart and blood vessels, leading to disruption in heart rate control and vascular dynamics ${ }^{1)}$ and resulting in increased mortality with a relative mortality risk of 3.5 (95\% confidence interval, 2.7-4.5). ${ }^{2}$ This can be explained by the increased rate of cardiac arrhythmias due to sympatho-vagal imbalance, ${ }^{3)}$ as well as to cardiac sympathetic denervation. ${ }^{4)}$ Therefore, diabetic patients must be closely supervised for early detection of CAN.

Since the early 1970s, the Ewing method has been widely used to diagnose CAN. ${ }^{5}$ In 2005, the American Diabetes Association consensus statement recommended a battery of cardiovascular reflex tests to detect cardiovascular autonomic dysfunction. ${ }^{6}$ Analysis of heart rate variability (HRV) has also been useful for detecting CAN in its early subclinical stage, ${ }^{\text {,) }}$ and reduced HRV has been considered an early warning sign. ${ }^{8)}$

The reported prevalence of CAN has varied depending on detection methodology used and characteristics of the study population, ${ }^{6,9)}$ Abnormal cardiovascular reflexes were 
detected in $7.7 \%$ of patients with type 1 diabetes mellitus (T1DM) at the time of diagnosis in a study by Ziegler et al., ${ }^{10)}$ in $13 \%$ of T1DM patients with more than 9 years of followup (mean diabetic duration of 14.7 years) in the Diabetes Control and Complications Trial (DCCT) study, ${ }^{11)}$ and in 36\% in T1DM patients with a mean diabetic duration of 15 years according to the EURODIAB IDDM Complications Study. ${ }^{12)}$ The risk of T1DM patients developing CAN is affected by several risk factors including age, sex, body mass index (BMI), duration of diabetes, glycemic control, cardiovascular risk profile (hyperlipidemia, hypertension), and microvascular complications (e.g., retinopathy, nephropathy), ${ }^{9,12-17)}$

In the present study, we evaluated the prevalence of CAN using standard cardiovascular reflex tests according to recent guidelines. We also evaluated the risk factors associated with reduced HRV in nonobese young adult T1DM patients without other micro- or macrovascular complications.

\section{Materials and methods}

\section{Subjects}

Patients with T1DM (18-29 years of age) receiving multiple daily injections of insulin were recruited from the outpatient clinic at Seoul National University Children's Hospital between January 2014 and January 2016. T1DM was defined by presence of one or more islet autoantibodies with low or undetectable level of plasma C-peptide. ${ }^{18)}$ Among the 126 patients with T1DM, those who declined to provide consent $(n=3)$, those with $<5$ years duration of diabetes $(n=2)$, diabetic retinopathy ( $\geq 1$ microaneurysm or hemorrhage) $(n=4)$, diabetic nephropathy (>30 $\mathrm{mg}$ of albumin in a 24-hour urine sample) $(\mathrm{n}=9)$, obesity $\left(\mathrm{BMI} \geq 25 \mathrm{~kg} / \mathrm{m}^{2}\right)(\mathrm{n}=8)$, cardiac arrhythmia $(\mathrm{n}=1)$, and prescription for antihypertensive medications $(n=4)$ were excluded. A final total of 95 patients was included in the study.

\section{Measurements}

Height was measured with a Harpenden stadiometer (Holtain Ltd., Crymych, Wales, UK), and weight was measured with a digital scale (150 A; Cas Co., Ltd., Seoul, Korea). BMI was calculated as weight $(\mathrm{kg})$ divided by height squared $\left(\mathrm{m}^{2}\right)$. Waist circumference was also measured. Body composition was analyzed by bioimpedance (InBody 770, InBody Co., Seoul, Korea). After a 10-minute rest, blood pressure (BP) was measured at the time of cardiovascular reflex testing using DiCAN (Medicore, Seoul, Korea).

The urinary albumin level was measured at baseline from a single 24-hour urine collection. Diabetic retinopathy was assessed from retinal photographs at baseline, and the findings were reviewed by a single ophthalmologist. Peripheral neuropathy was screened by a questionnaire, the Michigan Neuropathy Screening Instrument, ${ }^{19)}$ and tested by vibration threshold using a 128-Hz tuning fork and light touch perception using a $10 \mathrm{~g}$ (5.07 Semmes-Weinstein) monofilament. ${ }^{20)}$
Questionnaires regarding smoking history, family history of cardiovascular diseases, and symptoms of autonomic dysfunction (Abbreviated Composite Autonomic Symptom Score; COMPASS 31$)^{21)}$ were investigated.

Blood samples were obtained under conditions of metabolic stability, defined as absence of episodes of diabetic ketoacidosis during the previous month and hypoglycemic events the night before cardiovascular reflex testing. Using commercially available reagents, we measured the circulating levels of hemoglobin $\mathrm{A}_{1 \mathrm{c}}\left(\mathrm{Hb}_{\mathrm{cc}}\right)$, total cholesterol, high-density lipoprotein (HDL) cholesterol, and low-density lipoprotein cholesterol using a chemistry autoanalyzer (TBA-c16000 Automatic Analyzer, Toshiba, Tokyo, Japan). Mean $\mathrm{HbA}_{1} \mathrm{c}$ level during follow-up was calculated for each patient as an indicator of long-term glycemic control. Non-HDL cholesterol was calculated by subtracting HDL cholesterol from total cholesterol.

\section{Cardiac autonomic function testing}

Twenty-four hours prior to their visit, all participants were asked to refrain from heavy physical exercise, smoking, consumption of caffeinated beverages, and any medication that could influence cardiac autonomic function. Prior to cardiac autonomic function tests, glucose level was checked, and no patient was found to have hypoglycemia before or during the tests. Measurements were performed over a 5-minute period in the supine position after a 10-minute period of rest and heart rate stabilization. CAN was assessed by standard cardiovascular reflex tests as described by Ewing and Clarke. ${ }^{5,22)}$ Changes in $\mathrm{R}-\mathrm{R}$ with deep breathing, standing, and the Valsalva maneuver, as well as change in BP in response to standing were evaluated. The R-R difference with deep breathing was the mean of the difference between maximum and minimum R-R intervals during 6 respiratory cycles. The ratio of the longest R-R interval around the 30th beat after standing to the shortest R-R interval around the 15th beat after standing was 30:15. The Valsalva ratio was the ratio of longest $\mathrm{R}-\mathrm{R}$ interval after the maneuver to shortest $\mathrm{R}-\mathrm{R}$ interval during forced exhalation into the mouthpiece of a manometer to $40 \mathrm{mmHg}$ for 15 seconds. The response to cardiovascular reflex tests was categorized as either normal or abnormal. An R-R difference $\leq 10$ beats/min, a 30:15 ratio $\leq 1.0$, a Valsalva ratio $\leq 1.10,{ }^{5}$ and a decrease in $\mathrm{BP} \geq 20$ mmHg upon standing ${ }^{23)}$ were defined as abnormal. CAN was defined as at least one abnormal result out of the 4 tests. Spectral analyses of HRV (standard deviation of all normal R-R intervals $[\mathrm{SDNN}]$ and total power $[\mathrm{TP}]$ ) were also evaluated using DiCAN (Medicore). SDNN and TP are measurements of the overall HRV. Therefore, lower SDNN or TP level indicates reduced overall $\mathrm{HRV}^{7)}$

\section{Statistical analysis}

IBM SPSS Statistics ver. 22.0 (IBM Co., Armonk, NY, USA) was used for statistical analyses. All continuous variables 
were described as mean \pm standard deviation. Logarithmic transformations were performed before statistical analyses for variables that were not normally distributed. Independent sample $t$-tests were used to compare means of continuous variables. Chi-square tests were performed to determine the significance of categorical variables. Univariate and multivariate regression analyses were performed to examine the associations between HRV, as a continuous outcome, and clinical explanatory variables. Statistical significance was defined as $P<0.05$.

\section{Results}

\section{Characteristics of the study population}

Baseline characteristics of the 95 patients ( 45 males) are shown in Table 1. The mean age of T1DM patients was $22.2 \pm 2.9$ years (range, 18.0-29.5 years), and mean disease duration was $13.1 \pm 4.8$ years (range, $5.1-25.4$ years). The mean $\mathrm{HbA}_{1 \mathrm{c}}$ level was $8.4 \% \pm 1.2 \%$. Seventy-four patients $(77.9 \%)$ reported family history of cardiovascular disease. The mean $\mathrm{HbA}_{1 \mathrm{c}}$ level, fat mass percentage, and total cholesterol level were higher in women compared to men $(P<0.05)$. No patients exhibited clinical manifestations of peripheral neuropathy or CAN.

\section{Prevalence of CAN in nonobese T1DM patients without micro- or macrovascular complications}

Twelve patients (12.6\%) were diagnosed with CAN, which was defined as having $1(n=10,10.5 \%)$ or $2(n=2,2.1 \%)$ abnormal reflex test results (Table 1). No difference was found between the sexes in prevalence of CAN. The heart rate response to the Valsalva maneuver exhibited the most frequently abnormal results (7.4\%) among the 4 cardiovascular reflex tests, followed by heart rate response during deep breathing (4.2\%) and standing (3.2\%). No patient had an abnormal BP response to standing. Moreover, no difference was observed in overall HRV parameters (both SDNN and TP) between the sexes.

When clinical characteristics and cardiac autonomic function testing were compared with respect to presence of CAN, no significant differences were found in age, sex, mean $\mathrm{HbA}_{10}$ level, or duration of diabetes between patients with and those without CAN. Patients with CAN exhibited lower overall HRV parameters (both SDNN and TP) compared to those without CAN; however, the difference was not statistically significant (Table 2).

\section{Risk factors associated with decrease in overall HRV}

When we performed linear regression analysis to determine risk factors associated with overall HRV parameters, HRV was inversely associated with mean $\mathrm{HbA}_{1 \mathrm{c}}$ level $(P<0.001$ for

Table 1. Clinical characteristics of the study subjects

\begin{tabular}{|c|c|c|c|}
\hline Characteristic & Total $(n=95)$ & Male $(n=45)$ & Female $(n=50)$ \\
\hline Age (yr) & $22.2 \pm 2.9$ & $22.5 \pm 3.1$ & $22.0 \pm 2.7$ \\
\hline Duration of diabetes (yr) & $13.1 \pm 4.8$ & $13.8 \pm 5.5$ & $12.4 \pm 4.0$ \\
\hline $\mathrm{HbA}_{1 \mathrm{c}}(\%)$ & $8.4 \pm 1.2$ & $8.1 \pm 1.1$ & $8.7 \pm 1.2^{*}$ \\
\hline Body mass index $\left(\mathrm{kg} / \mathrm{m}^{2}\right)$ & $21.8 \pm 2.3$ & $22.3 \pm 2.1$ & $21.4 \pm 2.4$ \\
\hline Fat mass (\%) & $23.7 \pm 7.2$ & $17.7 \pm 4.8$ & $28.6 \pm 4.8^{*}$ \\
\hline Total cholesterol (mg/dL) & $190.9 \pm 34.4$ & $178.2 \pm 34.4$ & $202.3 \pm 28.7^{*}$ \\
\hline Non-HDL cholesterol (mg/dL) & $119.3 \pm 31.9$ & $114.0 \pm 34.4$ & $124.1 \pm 29.0$ \\
\hline $\mathrm{HR}(\mathrm{bpm})$ & $74.6 \pm 9.4$ & $75.2 \pm 9.9$ & $74.1 \pm 9.0$ \\
\hline Systolic blood pressure (mmHg) & $109.7 \pm 10.4$ & $116.1 \pm 9.8$ & $103.9 \pm 7.1^{*}$ \\
\hline Diastolic blood pressure (mmHg) & $70.5 \pm 6.9$ & $72.9 \pm 7.5$ & $68.2 \pm 5.6^{*}$ \\
\hline Family history of cardiovascular diseases & $74(77.9)$ & $36(80.0)$ & $38(76.0)$ \\
\hline \multicolumn{4}{|l|}{ Abnormal cardiovascular reflex tests } \\
\hline HR response to the Valsalva maneuver & $7(7.4)$ & $3(6.7)$ & $4(8.0)$ \\
\hline HR variability during deep breathing & $4(4.2)$ & $2(4.4)$ & $2(4.0)$ \\
\hline HR response to standing & $3(3.2)$ & $3(6.7)$ & $0(0)$ \\
\hline Blood pressure response to standing & $0(0)$ & $0(0)$ & $0(0)$ \\
\hline Cardiac autonomic neuropathy $^{\dagger}$ & $12(12.6)$ & $7(15.6)$ & $5(10.0)$ \\
\hline One abnormal reflex test & $10(10.5)$ & $6(13.3)$ & $4(8.0)$ \\
\hline Two abnormal reflex test & $2(2.1)$ & $1(2.2)$ & $1(2.0)$ \\
\hline \multicolumn{4}{|l|}{ Overall HR variability } \\
\hline SDNN (msec) & $41.1 \pm 16.2$ & $40.0 \pm 17.2$ & $42.2 \pm 15.3$ \\
\hline Total power $\left(\mathrm{msec}^{2}\right)$ & $1,438.9 \pm 1,222.0$ & $1,313.6 \pm 1,237.7$ & $1,551.6 \pm 1,209.1$ \\
\hline
\end{tabular}

Values are mean \pm standard deviation or number (\%).

$\mathrm{HbA}_{1 c}$, hemoglobin $\mathrm{A}_{1 c} ; \mathrm{HDL}$, high-density lipoprotein; HR, heart rate; SDNN, standard deviation of mean NN intervals.

${ }^{*} P<0.05$. ${ }^{\dagger}$ Cardiac autonomic neuropathy was defined as at least one abnormal result out of the 4 cardiovascular reflex tests 
Table 2. Comparison of clinical characteristics and cardiac autonomic function test according to the presence of CAN

\begin{tabular}{|c|c|c|c|}
\hline Variable & Non-CAN $(n=83)$ & CAN $(n=12)$ & $P$-value \\
\hline Age (yr) & $22.3 \pm 2.9$ & $21.9 \pm 3.3$ & 0.660 \\
\hline Male sex & $38(45.8)$ & $7(58.3)$ & 0.416 \\
\hline Duration of diabetes (yr) & $13.2 \pm 4.8$ & $12.0 \pm 4.6$ & 0.409 \\
\hline $\mathrm{HbA}_{1 c}(\%)$ & $8.4 \pm 1.2$ & $8.3 \pm 1.1$ & 0.898 \\
\hline Body mass index $\left(\mathrm{kg} / \mathrm{m}^{2}\right)$ & $21.8 \pm 2.3$ & $22.2 \pm 1.8$ & 0.562 \\
\hline Fat mass (\%) & $23.7 \pm 7.4$ & $23.6 \pm 6.5$ & 0.992 \\
\hline Total cholesterol (mg/dL) & $190.3 \pm 34.5$ & $194.8 \pm 35.1$ & 0.577 \\
\hline Non-HDL cholesterol (mg/dL) & $118.8 \pm 31.6$ & $122.9 \pm 34.7$ & 0.669 \\
\hline Heart rate (beats per minute) & $75.7 \pm 10.2$ & $74.2 \pm 9.2$ & 0.483 \\
\hline Systolic blood pressure (mmHg) & $109.3 \pm 10.6$ & $111.9 \pm 9.3$ & 0.388 \\
\hline Diastolic blood pressure (mmHg) & $70.4 \pm 7.1$ & $71.2 \pm 5.8$ & 0.798 \\
\hline SDNN (msec) & $41.7 \pm 16.0$ & $37.4 \pm 17.4$ & 0.391 \\
\hline Total power $\left(\mathrm{msec}^{2}\right)$ & $1,454.2 \pm 1,198.8$ & $1,333.3 \pm 1,426.5$ & 0.554 \\
\hline
\end{tabular}

Values are mean \pm standard deviation or number (\%).

CAN, cardiac autonomic neuropathy; $\mathrm{HbA}_{1 c}$, hemoglobin $\mathrm{A}_{1 c ;} \mathrm{HDL}$, high-density lipoprotein; SDNN, standard deviation of mean NN intervals.

Table 3. Multivariate-adjusted model to find risk factors associated with overall heart rate variability

\begin{tabular}{lccccc}
\hline \multirow{2}{*}{ Variable } & \multicolumn{2}{c}{ SDNN } & & \multicolumn{2}{c}{ Total power } \\
\cline { 2 - 3 } \cline { 5 - 6 } & $\beta \pm$ SE & $P$-value & & $\beta \pm S E$ & $P$-value \\
\hline Age & $-1.22 \pm 0.64$ & 0.060 & & $-0.09 \pm 0.03$ & 0.011 \\
Female vs. male & $6.42 \pm 3.24$ & 0.051 & & $0.58 \pm 0.17$ & 0.001 \\
Disease duration & $1.64 \pm 4.94$ & 0.741 & & $0.41 \pm 0.27$ & 0.129 \\
HbA $_{1 c}$ & $-44.42 \pm 13.76$ & 0.002 & & $-2.82 \pm 0.74$ & $<0.001$ \\
$\begin{array}{l}\text { Body mass index } \\
\left(\mathrm{kg} / \mathrm{m}^{2}\right)\end{array}$ & $0.74 \pm 0.72$ & 0.306 & & $0.08 \pm 0.04$ & 0.035 \\
Non-HDL cholesterol & $-9.75 \pm 6.98$ & 0.166 & & $-0.45 \pm 0.37$ & 0.233 \\
\hline
\end{tabular}

Values are presented as regression coefficient $(\beta) \pm$ standard error (SE).

SDNN, standard deviation of mean NN intervals; $\mathrm{HbA}_{1 c}$, hemoglobin $A_{1 c ;} H D L$, high-density lipoprotein.

both SDNN and TP) and non-HDL cholesterol $(P=0.002$ for SDNN, and $P=0.013$ for TP). After adjusting for all possible covariates including age, sex, duration of diabetes, mean $\mathrm{HbA}_{1 \mathrm{c}}$, $\mathrm{BMI}$, and non-HDL-cholesterol, higher mean $\mathrm{HbA}_{\mathrm{Ic}}$ level was significantly associated with lower overall HRV $(\beta=-44.42$, $P=0.002$ for SDNN and $\beta=-2.82, P<0.001$ for TP) (Table 3 ).

\section{Discussion}

In our study, CAN was detected in 12.6\% of nonobese T1DM patients aged 18 to 29 years without micro- or macrovascular complications. Patients with CAN tended to have lower overall HRV (SDNN and TP). In addition, overall HRV was significantly reduced in the presence of poor glycemic control.

Since CAN is a serious complication associated with mortality in diabetic patients, ${ }^{2,24)}$ possible symptoms and signs related to CAN should not be overlooked in T1DM patients with disease duration $\geq 5$ years. ${ }^{6}$ Therefore, in 2011, the Toronto Consensus Panel on Diabetic Neuropathy recommended use of cardiovascular reflex tests measuring end-organ response (heart rate and $\mathrm{BP}$ changes) during provocative physiological maneuvers such as heart rate response to deep breathing, standing, and the Valsalva maneuver and BP response to standing. ${ }^{22)}$ One abnormal test was defined as early CAN; at least 2 abnormal tests were defined as definite CAN. ${ }^{22,25)}$

The prevalence of CAN in T1DM has been reported with wide variations from $1.6 \%$ to $90 \%$ depending on diagnostic criteria used and study population. ${ }^{6.9)}$ Our results are consistent with the $13 \%$ prevalence among 1,441 T1DM patients (mean age of $26.5 \pm 7.0$ years) with more than 9 years of follow-up in the DCCT study, ${ }^{11)}$ which defined CAN as at least one abnormal result of 3 autonomic tests ( $\mathrm{R}-\mathrm{R}$ variation with paced breathing, Valsalva ratio, and postural BP changes). The EURODIAB IDDM Complications Study reported a prevalence rate of 36\% in 3,007 T1DM patients (mean age of $32.7 \pm 10.2$ years, mean duration of diabetes $14.7 \pm 9.3$ years) when CAN was defined as abnormal results for at least one of 2 cardiovascular reflex tests (change in heart rate and BP on standing). ${ }^{12}$ In the Pittsburgh Epidemiology of Diabetes Complications Study, 168 T1DM patients (mean age of 29 years, mean duration of diabetes 20 years) were evaluated by heart rate responses to deep breathing, standing, and the Valsalva maneuver; $37.5 \%$ of the patients were diagnosed with CAN (at least 1 abnormal test out of 3). ${ }^{26} \mathrm{~A}$ higher prevalence of CAN was seen in previous studies resulting from inclusion of adult patients who had long-term follow-up and/or micro- or macrovascular complications. ${ }^{12,26)}$ In 2011 , the Toronto Consensus Panel recommended CAN screening for TIDM patients after 5 years of disease, particularly in those with poor glycemic control. ${ }^{22)}$ Our study showed that nonobese young adults with T1DM can be diagnosed with earlystage CAN despite the absence of micro- or macrovascular complications, necessitating consideration for CAN screening followed by history taking of suspected symptoms.

The overall HRV parameters $(41.1 \pm 16.2$ for SDNN, $1,438.9 \pm 1,222.0$ for TP) in our T1DM patients were lower than those for the same age group in a previously published report in 
healthy Koreans $\left(51.9 \pm 57.3\right.$ for SDNN, 1973.0 \pm 1723.1 for TP), ${ }^{27)}$ even though this study was limited by lack of a comparison with healthy control subjects. Previous studies have demonstrated reduced overall HRV in patients with T1DM compared to control subjects. ${ }^{28,29)}$ Since early subclinical CAN could be detected by power spectral analyses of $\mathrm{HRV}^{30)}$ analyses of shortterm HRV may help identify individuals at risk for unexpected mortality related to $\mathrm{CAN}$. $^{31}$

In the present study, poor glycemic control was significantly related to reduced HRV even though no significant difference was found in $\mathrm{HbA}_{1 \mathrm{c}}$ level between patients with CAN and those without due to small sample size and small number of patients diagnosed with CAN. The Framingham Heart Study reported an inverse relationship between HRV and blood glucose level in a large community-based population. ${ }^{32)}$ In newly diagnosed T1DM patients, intensive glycemic control had a beneficial effect on prevention of reduced HRV during 24 years of followup. ${ }^{33)}$ The DCCT study also reported that intensive glycemic control can prevent and slow progression of CAN. ${ }^{11)}$ Prolonged hyperglycemia is believed to affect myelination of autonomic nerves by activating various biochemical pathways causing tissue hypoxia, increased free oxygen radicals, and formation of advanced glycation end products. ${ }^{34)}$

A recent longitudinal study in old patients with type 2 diabetes reported BMI and higher $\mathrm{HbA}_{1 \mathrm{c}}$ as being associated with lower HRV indices even though the effects diminished over time. ${ }^{35)}$ However, BMI was not related to reduced HRV in nonobese, young adults with T1DM in our study. Poor glycemic control is a common risk factor for CAN in both types of diabetes (types 1 and 2), whereas several cardiovascular risk factors (obesity, dyslipidemia, and hypertension) are more important risk factors of CAN in type 2 diabetes patients. ${ }^{22)}$

Although duration of diabetes has been suggested as one of the risk factors of $\mathrm{CAN},{ }^{11,12,16,17,36)}$ some studies have reported no significant association, ${ }^{37,38)}$ as seen in the present study. The lack of association between diabetes duration and CAN in our study may be explained by the inclusion criteria. We determined whether CAN was present in young adults with T1DM, aged 18 to 29 years, without micro- or macrovascular complications. Although this study included some diabetic patients with a duration $>10$ years, the duration after puberty may have been relatively short compared to that in previous adult studies. As patients were older, longer diabetes duration may lead to chronic complications including CAN. Therefore, long-term follow-up studies are warranted in a large population.

This study was limited by its cross-sectional design and small sample size. Therefore, we were unable to provide statistical significance among the risk factors of CAN in our study subjects. However, this study was strengthened by use of standard cardiovascular reflex tests as recommended by current guidelines and evaluation of HRV parameters in young, nonobese T1DM adults without micro- or macrovascular complications.

In conclusion, CAN was detected in $12.6 \%$ of nonobese young adult T1DM patients without micro- or macrovascular complications, which supports the necessity of CAN screening even in young adults without these complications. Considering the relationship between poor glycemic control and reduced overall HRV, we suggest that good glycemic control is mandatory for maintaining overall HRV and preventing chronic complications related to diabetes. Further studies are needed to fully understand the natural course of CAN to determine the methods to reverse it in its early stages in young adults with T1DM.

\section{Ethical statement}

This study was approved by the Institutional Review Board at Seoul National University Hospital (IRB No. H-1311-078535). Written informed consent was submitted by all enrolled subjects.

\section{Conflict of interest}

No potential conflict of interest relevant to this article was reported.

\section{Acknowledgments}

This work was supported by a Korean Society of Pediatric Endocrinology grant (Grant Number 2013-02) and the Seoul National University Hospital Research Fund (Grant Number 04-2013-3040).

\section{References}

1. Vinik AI, Erbas T, Casellini CM. Diabetic cardiac autonomic neuropathy, inflammation and cardiovascular disease. J Diabetes Investig 2013;4:4-18.

2. Maser RE, Mitchell BD, Vinik AI, Freeman R. The association between cardiovascular autonomic neuropathy and mortality in individuals with diabetes: a meta-analysis. Diabetes Care 2003;26:1895-901.

3. Kleiger RE, Miller JP, Bigger JT Jr, Moss AJ. Decreased heart rate variability and its association with increased mortality after acute myocardial infarction. Am J Cardiol 1987;59:256-62.

4. Stevens MJ, Raffel DM, Allman KC, Dayanikli F, Ficaro E, Sandford T, et al. Cardiac sympathetic dysinnervation in diabetes: implications for enhanced cardiovascular risk. Circulation 1998;98:961-8.

5. Ewing DJ, Clarke BF. Diagnosis and management of diabetic autonomic neuropathy. Br Med J (Clin Res Ed) 1982;285:916-8.

6. Boulton AJ, Vinik AI, Arezzo JC, Bril V, Feldman EL, Freeman R, et al. Diabetic neuropathies: a statement by the American Diabetes Association. Diabetes Care 2005;28:956-62. 
7. Heart rate variability. Standards of measurement, physiological interpretation, and clinical use. Task Force of the European Society of Cardiology and the North American Society of Pacing and Electrophysiology. Eur Heart J 1996;17:354-81.

8. Schönauer M, Thomas A, Morbach S, Niebauer J, Schönauer U, Thiele H. Cardiac autonomic diabetic neuropathy. Diab Vasc Dis Res 2008;5:336-44.

9. Tang M, Donaghue KC, Cho YH, Craig ME. Autonomic neuropathy in young people with type 1 diabetes: a systematic review. Pediatr Diabetes 2013;14:239-48.

10. Ziegler D, Gries FA, Spüler M, Lessmann F. The epidemiology of diabetic neuropathy. Diabetic Cardiovascular Autonomic Neuropathy Multicenter Study Group. J Diabetes Complications 1992;6:49-57.

11. The effect of intensive diabetes therapy on measures of autonomic nervous system function in the Diabetes Control and Complications Trial (DCCT). Diabetologia 1998;41:416-23.

12. Kempler P, Tesfaye S, Chaturvedi N, Stevens LK, Webb DJ, Eaton $\mathrm{S}$, et al. Autonomic neuropathy is associated with increased cardiovascular risk factors: the EURODIAB IDDM Complications Study. Diabet Med 2002;19:900-9.

13. Serhiyenko VA, Serhiyenko AA. Cardiac autonomic neuropathy: risk factors, diagnosis and treatment. World J Diabetes 2018;9:1-24.

14. Dafaalla MD, Nimir MN, Mohammed MI, Ali OA, Hussein A. Risk factors of diabetic cardiac autonomic neuropathy in patients with type 1 diabetes mellitus: a meta-analysis. Open Heart 2016;3:e000336.

15. Witte DR, Tesfaye S, Chaturvedi N, Eaton SE, Kempler P, Fuller JH, et al. Risk factors for cardiac autonomic neuropathy in type 1 diabetes mellitus. Diabetologia 2005;48:164-71.

16. Stella P, Ellis D, Maser RE, Orchard TJ. Cardiovascular autonomic neuropathy (expiration and inspiration ratio) in type 1 diabetes. Incidence and predictors. J Diabetes Complications 2000;14:1-6.

17. Orchard TJ, LLoyd CE, Maser RE, Kuller LH. Why does diabetic autonomic neuropathy predict IDDM mortality? An analysis from the Pittsburgh Epidemiology of Diabetes Complications Study. Diabetes Res Clin Pract 1996;34 Suppl:S165-71.

18. American Diabetes Association. 2. Classification and diagnosis of diabetes: standards of medical care in diabetes-2019. Diabetes Care 2019;42(Suppl 1):S13-28.

19. Herman WH, Pop-Busui R, Braffett BH, Martin CL, Cleary PA, Albers JW, et al. Use of the Michigan Neuropathy Screening Instrument as a measure of distal symmetrical peripheral neuropathy in Type 1 diabetes: results from the Diabetes Control and Complications Trial/Epidemiology of Diabetes Interventions and Complications. Diabet Med 2012;29:937-44.

20. Singh N, Armstrong DG, Lipsky BA. Preventing foot ulcers in patients with diabetes. JAMA 2005;293:217-28.
21. Sletten DM, Suarez GA, Low PA, Mandrekar J, Singer W. COMPASS 31: a refined and abbreviated Composite Autonomic Symptom Score. Mayo Clin Proc 2012;87:1196201 .

22. Spallone V, Ziegler D, Freeman R, Bernardi L, Frontoni S, Pop-Busui R, et al. Cardiovascular autonomic neuropathy in diabetes: clinical impact, assessment, diagnosis, and management. Diabetes Metab Res Rev 2011;27:639-53.

23. American Diabetes Association. 10. Microvascular complications and foot care: standards of medical care in diabetes-2018. Diabetes Care 2018;41(Suppl 1):S105-18.

24. Soedamah-Muthu SS, Chaturvedi N, Witte DR, Stevens LK, Porta M, Fuller JH, et al. Relationship between risk factors and mortality in type 1 diabetic patients in Europe: the EURODIAB Prospective Complications Study (PCS). Diabetes Care 2008;31:1360-6.

25. Spallone V, Bellavere F, Scionti L, Maule S, Quadri R, Bax G, et al. Recommendations for the use of cardiovascular tests in diagnosing diabetic autonomic neuropathy. Nutr Metab Cardiovasc Dis 2011;21:69-78.

26. Maser RE, Pfeifer MA, Dorman JS, Kuller LH, Becker DJ, Orchard TJ. Diabetic autonomic neuropathy and cardiovascular risk. Pittsburgh Epidemiology of Diabetes Complications Study III. Arch Intern Med 1990;150:121822.

27. Kim GM, Woo JM. Determinants for heart rate variability in a normal Korean population. J Korean Med Sci 2011;26:1293-8.

28. Jaiswal M, Urbina EM, Wadwa RP, Talton JW, D'Agostino RB Jr, Hamman RF, et al. Reduced heart rate variability among youth with type 1 diabetes: the SEARCH CVD study. Diabetes Care 2013;36:157-62.

29. Rosengård-Bärlund M, Bernardi L, Fagerudd J, Mäntysaari M, Af Björkesten CG, Lindholm H, et al. Early autonomic dysfunction in type 1 diabetes: a reversible disorder? Diabetologia 2009;52:1164-72.

30. Wawryk AM, Bates DJ, Couper JJ. Power spectral analysis of heart rate variability in children and adolescents with IDDM. Diabetes Care 1997;20:1416-21.

31. Tsuji H, Venditti FJ Jr, Manders ES, Evans JC, Larson MG, Feldman CL, et al. Reduced heart rate variability and mortality risk in an elderly cohort. The Framingham Heart Study. Circulation 1994;90:878-83.

32. Singh JP, Larson MG, O'Donnell CJ, Wilson PF, Tsuji H, Lloyd-Jones DM, et al. Association of hyperglycemia with reduced heart rate variability (The Framingham Heart Study). Am J Cardiol 2000;86:309-12.

33. Ziegler D, Behler M, Schroers-Teuber M, Roden M. Nearnormoglycaemia and development of neuropathy: a 24year prospective study from diagnosis of type 1 diabetes. BMJ Open 2015;5:e06559.

34. Pop-Busui R. Cardiac autonomic neuropathy in diabetes: a clinical perspective. Diabetes Care 2010;33:434-41.

35. Andersen ST, Witte DR, Fleischer J, Andersen H, Lauritzen $\mathrm{T}$, Jørgensen ME, et al. Risk factors for the presence and 
progression of cardiovascular autonomic neuropathy in type 2 diabetes: ADDITION-Denmark. Diabetes Care 2018;41:2586-94.

36. Orlov S, Cherney DZ, Pop-Busui R, Lovblom LE, Ficociello LH, Smiles AM, et al. Cardiac autonomic neuropathy and early progressive renal decline in patients with nonmacroalbuminuric type 1 diabetes. Clin J Am Soc Nephrol 2015;10:1136-44.
37. Straub RH, Zietz B, Palitzsch KD, Schölmerich J. Impact of disease duration on cardiovascular and pupillary autonomic nervous function in IDDM and NIDDM patients. Diabetes Care 1996;19:960-7.

38. Piha SJ, Halonen JP. Effect of disease duration on cardiac autonomic reflexes in young patients with insulindependent diabetes mellitus. Diabetes Res Clin Pract 1992;18:61-7. 